EPJ Web of Conferences 73, 05001 (2014)

DOI: $10.1051 /$ epjconf/20147305001

(C) Owned by the authors, published by EDP Sciences, 2014

\title{
Charmed hadrons in matter and SU(4) flavor symmetry
}

\author{
Gastão Krein ${ }^{\mathrm{a}}$ \\ Instituto de Físiva Teórica, Universidade Estadual Paulista Rua Dr. Bento Teobaldo Ferraz, \\ 271 - Bloco II, 01140-070 São Paulo, SP, Brazil
}

\begin{abstract}
There is great recent interest in the study of bound states of charmed hadrons with atomic nuclei. The studies rely on effective interactions expressed through couplings between charmed and light-flavored hadrons whose values are fixed using SU(4) flavor symmetry. In the present communication we present results of recent studies examining the accuracy of SU(4)-flavor symmetry relations between hadron-hadron couplings with particular interest in the couplings of charmed D mesons to light mesons and nucleons. We discuss results obtained from a ${ }^{3} P_{0}$ quark-pair creation model and from a framework based on Dyson-Schwinger equations in QCD that incorporates a consistent, direct and simultaneous description of light- and heavy-quarks. We focus on the three-meson couplings $\rho \pi \pi, \rho K K$, and $\rho D D$ and meson-baryon-brayon couplings $\pi N N, K \Lambda_{s} N$, and $D \Lambda_{c} N$. While the ${ }^{3} P_{0}$ model predicts that the SU(4) breaking is at most $40 \%$ in the charm sector, the relativistic Dyson-Schwinger framework predicts a breaking 10 times bigger. Consequences of these findings for the predictions of DN cross sections, formation of bound states of D-mesons and $J / \Psi$, and the formation of charmed hypernuclei are discussed.
\end{abstract}

\section{Charm in matter and the $D N$ interaction}

The study of the interaction of heavy-flavored hadrons with matter is of interest in different contexts. One instance of interest is related to the exciting perspective of creating new exotic nuclear bound states. Examples are $J / \Psi$ and $\eta_{c}$ forming bound states with a nucleus [1-5], charmed hypernuclei [6], and charmed heavy-light $D$ and $D^{*}$ mesons [7-9] - for a recent review, see Ref. [10]. A pre-requisite for reliable predictions of properties of charmed hadrons in matter is the understanding of their interactions with nucleons and other light mesons in free space. A particularly critical issue in the study of such exotic nuclear states is the $D N$ interaction. There is no direct experimental information available for this interaction and, for the time being, one has to rely on models constrained by symmetry, analogies with other similar processes, and the use of different degrees of freedom [11-17].

Effective Lagrangians based on SU(4)-flavor symmetry are widely used in the study of the interactions of charmed hadrons with matter. In the particular case of the exotic $J / \Psi$ nuclear bound state [2-5], the $J / \Psi$ meson interacts with the bound nucleons via virtual $D$ and $D^{*}$ loops - see Fig. 1 .

\footnotetext{
a e-mail: gkrein@ift.unesp.br
}

This is an Open Access article distributed under the terms of the Creative Commons Attribution License 4.0, which permits unrestricted use, distribution, and reproduction in any medium, provided the original work is properly cited. 

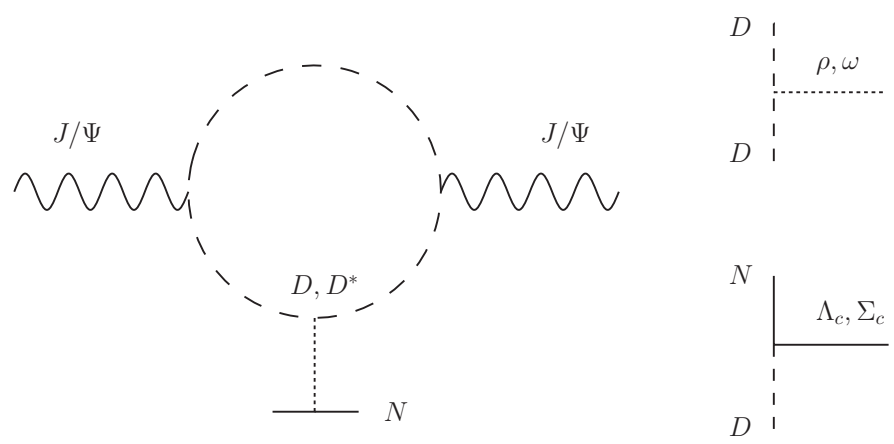

Figure 1. $J / \Psi$ in matter: the virtual $D$ and $D^{*}$ mesons interact with the nucleons of the medium. On the right, typical hadronic vertices that contribute to the $D N$ interaction.

In the absence of direct experimental information, the common strategy to obtain couplings like $g_{D D \rho}$ and $g_{N \Lambda_{c} D}$ is to use the $\mathrm{SU}(4)$ symmetry identities:

$$
\frac{g_{\pi \pi \rho}}{2}=g_{K K \rho}=g_{\bar{D} \bar{D} \rho} \quad \text { and } \quad g_{N N \pi}=g_{N \Lambda_{s} K}=g_{N \Lambda_{c} \bar{D}}
$$

where $g_{\pi \pi \rho}$ and $g_{N N \pi}$ are well constrained experimentally. Since flavor symmetry is badly broken by the quarks masses at the level of the QCD Lagrangian, $m_{u} \ll m_{s} \ll m_{c}$, an important issue is to what extent the breaking affects hadron-hadron couplings. The couplings are determined by transition matrix elements involving three hadron states. In terms of quark-gluon degrees of freedom, the matrix elements depend on the wave functions of the hadrons involved in the transition and as such depend on the quark masses. We start with the study conducted in the context of a ${ }^{3} P_{0}$ quark-pair creation model with hadron wave functions obtained from a nonrelativistic quark model (NRQM) [18]. The results obtained with the ${ }^{3} P_{0}$ model are then contrasted with those obtained in a framework using Dyson-Schwinger (DSE) equations, in which all elements are constrained by studies of QCD phenomenology, incorporating a consistent, direct and simultaneous description of light- and heavy-quarks [19].

\section{Hadron couplings in the ${ }^{3} P_{0}$ and DSE frameworks}

The matrix element of the hadronic transition $A \rightarrow B+C$ in the ${ }^{3} P_{0}$ model, defined in terms of paircreation Hamiltonian $H_{q \bar{q}}$, can be written as [18]

$$
\left\langle B C\left|H_{q \bar{q}}\right| A\right\rangle=\delta\left(\mathbf{P}_{A}-\mathbf{P}_{B}-\mathbf{P}_{C}\right) g_{A B C}\left(\mathbf{q}^{2}\right)|\mathbf{q}| Y_{11}(\hat{\mathbf{q}}),
$$

where $\mathbf{q}=\mathbf{P}_{B}-\mathbf{P}_{C}$ is the relative momentum between the final hadrons (the initial hadron is at rest), $F\left(\mathbf{q}^{2}\right)$ is a form factor describing the overlap of the three hadrons in the decay process, and $Y_{11}(\hat{\mathbf{q}})$ is a spherical harmonic function - only the $Y_{11}$ appears in the couplings discussed here. For the couplings in Eq. (1), hadrons $A$ and $C$ are the same.

To calculate the matrix element, one needs the wave functions of the hadrons calculated from the quark model Hamiltonian. We obtain the hadron wave functions using the quark model Hamiltonian and corresponding parameters of Ref. [20]. The wave functions of the mesons and baryons are found by diagonalizing the Hamiltonian in a finite basis of Gaussian wave functions with a common variational parameter; details will be presented elsewhere [18].

In the framework of the DSEs, the basic imputs are the propagators of the quarks and the BetheSalpeter amplitudes of the mesons - we have not calculated baryon couplings within this framework. Since the approach is fully covariant, the transition form factor $F_{A B C}$ is a function of the (Euclidean) four momentum $q^{2}$, instead of the three-momentum $|\mathbf{q}|^{2}$ as in the ${ }^{3} P_{0}$ model, Eq. (2). The calculation 
of $F_{A B C}\left(q^{2}\right)$ involves several interconnected steps [19]: (1) solution of the Dyson-Schwinger equation for the propagators of the light and heavy quarks; (2) with the the derived quark propagators, the meson bound-state amplitudes are obtained from solving Bethe-Salpeter equations - they are normalized such that the experimental values of masses and electroweak decays constants of the mesons are correctly reproduced; and, (3) the matrix element is obtained by evaluating a triangle graph involving the quark propagators and the Bethe-Salpeter amplitudes. Physically motivated and controlled approximations in some of the steps above were implemented, all tested in several previous studies using this approach discussion of the approximations and references to relevant work are given in Ref. [19].

\section{Numerical results}

The parameters entering the quark-model Hamiltonian are standard [20]. We have obtained the masses and wave-functions of $\pi, \rho, K, D, N, \Lambda$, and $\Lambda_{c}$; stable results are obtained with eleven basis Gaussian functions. The experimental masses for the mesons and light and strange baryons are well reproduced, and those for the charmed baryons are reproduced within 10\%. Once the hadron wave-functions are obtained, the matrix element in Eq. (2) can be readily calculated. Within the DSE framework, the numerical results for $g_{P P V}\left(q^{2}\right)$ on the domain $q^{2}=\left\{-m_{\rho}^{2},+m_{\rho}^{2}\right\}$ can be fitted by the formula

$$
g_{P P \rho}\left(q^{2}\right)=\frac{a_{P}-b_{P} q^{2}}{1+c_{P} q^{2}+d_{P} q^{4}},
$$

with the constants given by: $a_{\pi, K, D}=(1.84,0.94,5.05), b_{\pi, K, D}=(1.45,0.62,4.26) \mathrm{GeV}^{-2}, c_{\pi, K, D}=$ $(0.75,0.55,0.36) \mathrm{GeV}^{-2}$, and $d_{\pi, K, D}=(0.085,-0.16,-0.06) \mathrm{GeV}^{-4}$. Results are shown in Table 1.

Table 1. Ratios of coupling constants relevant for the $D N$ interaction. The $P P \rho$ are evaluated at the $\rho$ pole: $g_{P P \rho}\left(q^{2}=-m_{\rho}^{2}\right)$. The baryon-baryon-meson couplings are evaluated respectively at the poles of the exchanged baryon: $g_{N N \pi}\left(q^{2}=-m_{N}^{2}\right), g_{N \Lambda K}\left(q^{2}=-m_{\Lambda}^{2}\right)$, and $g_{N \Lambda_{c} D}\left(q^{2}=-m_{\Lambda_{c}}^{2}\right)$.

\begin{tabular}{lccccccc}
\hline Model & $\frac{g_{\pi \pi \rho}}{2 g_{K K \rho}}$ & $\frac{g_{\pi \pi \rho}}{2 g_{\bar{D} \bar{D} \rho}}$ & $\frac{g_{K K \rho}}{g_{\bar{D} \bar{D} \rho}}$ & $\mid$ & $\frac{g_{N N \pi}}{g_{N \Lambda_{s} K}}$ & $\frac{g_{N N \pi}}{g_{N \Lambda_{c} \bar{D}}}$ & $\frac{g_{N \Lambda_{s} K}}{g_{N \Lambda_{c} \bar{D}}}$ \\
\hline SU(4) & 1 & 1 & 1 & $\mid$ & 1 & 1 & 1 \\
${ }^{3} P_{0}$ & 0.99 & 1.10 & 1.11 & $\mid$ & 1.09 & 0.60 & 0.70 \\
DSE & 0.98 & 0.23 & 0.21 & | & - & - & - \\
\hline
\end{tabular}

The results indicate a small violation of the SU(4) identities when going from the light-flavor $u, d$ sector to the strange $s$ sector: the violation is of the order of $1-2 \%$ in $g_{P P \rho}$ in both ${ }^{3} P_{0}$ and DSE approaches, and of the order of $10 \%$ in the baryon sector. The situation is very different when one moves to the charm sector. In the ${ }^{3} P_{0}$ approach, the violation in $g_{P P \rho}$ is of the order of $10 \%$, and in the baryon sector it can reach $40 \%$. In the DSE approach, the violation in $g_{P P \rho}$ is very large, of the order of $400 \%$. An interesting feature here is that the trend of breaking is different in both approaches; while in the ${ }^{3} P_{0}$ model the breaking is in the direction of given a smaller coupling for the charmed mesons, the DSE approach predicts that these couplings increase.

\section{Summary and perspectives}

At first sight, such large violations of SU(4) symmetry could indicate dramatic consequences for the $D N$ interactions and also the phenomenology of exotic charmed nuclei. However, as remarked in Ref. [19], the form factors encountered here are much softer as those used in meson exchange models, e.g. in Refs. $[11,12]$, so that the product of a large coupling and a soft form factor leads to an inflated $\bar{D} N$ cross-section by a factor of 5 only. 
Future work intends to clarify the reasons for the discrepancy of the predictions of the two approaches discussed here. The consideration of charmed hypernuclei [6], where $\Lambda_{c}$ and $\Sigma_{c}$ baryons are implanted in a nucleus, is an interesting new direction that can be explored experimentally at FAIR in Germany and JPARC in Japan. In addition to strong couplings like the ones discussed here, weak decay constants will be required to describe nonmesonic decay of such nuclei [21]. A nonmesonic decay is a unique signature of the formation of a charmed hypernucleus, as it can only happen because the $\Lambda_{c}$ or $\Sigma_{c}$ interact with nucleons in a nucleus. The interaction of $D$ mesons with nucleons is another important ingredient in these decays.

Work partially financed by Fundação de Amparo à Pesquisa do Estado de São Paulo - FAPESP, Grant Nos. 2009/50180-0 and 2013/01907-0, and Conselho Nacional de Desenvolvimento Científico e Tecnológico - CNPq, Grant No. 305894/2009-9.

\section{References}

[1] S.J. Brodsky, I.A. Schmidt, and G.F. de Teramond, Phys. Rev. Lett. 64, 1011 (1990)

[2] S.H. Lee and C.M. Ko, Phys. Rev. C 67, 038202 (2003)

[3] G. Krein, A.W. Thomas and K. Tsushima, Phys. Lett. B 697, 136 (2011)

[4] K. Tsushima, D.H. Lu, G. Krein and A.W. Thomas, Phys. Rev. C 83, 065208 (2011)

[5] G. Krein, J. Phys. Conf. Ser. 422, 012012 (2013)

[6] C.B. Dover and S.H. Kahana, Phys. Rev. Lett. 39, 1506 (1977)

[7] K. Tsushima, D.H. Lu, A.W. Thomas, K. Saito, and R.H. Landau, Phys. Rev. C 59, 2824 (1999)

[8] C. Garcia-Recio, J. Nieves and L. Tolos, Phys. Lett. B 690, 369 (2010)

[9] C. Garcia-Recio, J. Nieves, L.L. Salcedo and L. Tolos, Phys. Rev. C 85, 025203 (2012)

[10] L. Tolos, Int. J. Mod. Phys. E 22, 1330027 (2013)

[11] J. Haidenbauer, G. Krein, U.-G. Meißner, and A. Sibirtsev, Eur. Phys. J. A 33, 107 (2007)

[12] J. Haidenbauer, G. Krein, U.-G. Meissner and A. Sibirtsev, Eur. Phys. J. A 37, 55 (2008)

[13] J. Haidenbauer and G. Krein, Phys. Lett. B 687, 314 (2010)

[14] J. Haidenbauer, G. Krein, U.-G. Meissner and L. Tolos, Eur. Phys. J. A 47, 18 (2011)

[15] C.E. Fontoura and G. Krein, Few Body Syst. 50, 219 (2011)

[16] C.E. Fontoura, G. Krein and V.E. Vizcarra, EPJ Web Conf. 3, 03027 (2010)

[17] C.E. Fontoura, G. Krein and V.E. Vizcarra, Phys. Rev. C 87, 025206 (2013)

[18] C.E. Fontoura, J. Haidenbauer and G. Krein, in preparation

[19] B. El-Bennich, G. Krein, L. Chang, C.D. Roberts and D.J. Wilson, Phys. Rev. D 85, 031502 (2012)

[20] E.S. Swanson, Annals Phys. 220, 73 (1992)

[21] A.P. Galeão, S. Ghosh, C.E. Fontoura, G. Krein and F. Krmpotic, work in progress 